\title{
Some Properties of Fundamental Linear Canonical Zak Transform
}

\author{
Asriadi $^{1}$, Budi Nurwahyu' ${ }^{2}$, Mawardi Bahri ${ }^{3}$
}

\begin{abstract}
In this paper, we introduce some fundamentally properties of Zak linear canonical transform (LCZT) such as linearity and translation properties. LCZT is developing of Zak transform ( ZT) and linear canonical transform (LCT).
\end{abstract}

Keywords: linear canonical Zak transform; Zak transform; linear canonical transform; linearity property; translation property.

\begin{abstract}
Abstrak
Dalam jurnal ini akan diungkapkan beberapa sifat fundamental dari transformasi Zak linear kanonik (LCZT), yaitu sifat linear dan sifat translasi. LCZT merupakan hasil pengembangan dari dua buah transformasi yaitu transformasi Zak (ZT) dan transformasi linear kanonik (LCT).
\end{abstract}

Kata kunci:Transformasi Zak linear kanonik; transformasi Zak; transformasi linear kanonik; sifat linear; sifattranslasi.

\section{Introduction} follows.

Definition of Zak transformation was be introduced in 1967 by J. Zak [4] that stated as

\section{Definisi1.1[3,4]}

Zak transformation $Z f(t, \omega)$ of a function $f(t) \in L^{2}(\mathbb{R})$ is defined as

$\tilde{f}(t, \omega)=\left(Z_{a} f\right)(t, \omega)=\sqrt{a} \sum_{k \in \mathbb{Z}} f(a t-a k) e^{-2 \pi i k \omega}$

where $a>0$ and $t, \omega$ be real number parameters.

\footnotetext{
${ }^{I}$ Pascasarjana Mat14ematika Terapan, Fakultas MIPA,Universitas Hasanuddin, ${ }^{1}$ asriadiasrunmath@gmail.com

${ }^{2,3}$ Program studi matematika, Fakultas MIPA,Universitas Hasanuddin 2budinurwahyu@gmail.com, ${ }^{3}$ mawardibahri@gmail.com
} 


\section{Asriadi $^{1}$, Budi Nurwahyu ${ }^{2}$, Mawardi Bahri ${ }^{3}$}

Without loss of generality, by taking $a=1$ then from (1.1) we get $(Z f)(t, \omega)=\sum_{k \in \mathbb{Z}} f(t-k) e^{-2 \pi i k \omega}$.

Some properties that hold in Zak transformation are given as follows

\section{Theorem 1.1[3,4]}

1. Linearity.

Zak transformation is linear, namely for real number $a$ and $b$ this equation holds

$$
[Z(a f+b g)](t, \omega)=a \tilde{f}(t, \omega)+b \tilde{g}(t, \omega) .
$$

2. Translation.

$$
\left.\begin{array}{c}
{\left[\mathcal{Z}\left(T_{a} f\right)\right](t, \omega)=\tilde{f}(t-a, \omega),} \\
{\left[\mathcal{Z}\left(T_{-m} f\right)\right](t, \omega)=e^{2 \pi i m \omega} \tilde{f}(t, \omega)}
\end{array}\right] .
$$

3. Modulation.

$$
\begin{gathered}
{\left[Z\left(M_{b} f\right)\right](t, \omega)=e^{i b t} \tilde{f}\left(t, \omega-\frac{b}{2 \pi}\right),} \\
{\left[Z\left(M_{2 \pi b} f\right)\right](t, \omega)=e^{2 \pi i b t} \tilde{f}(t, \omega-b) .}
\end{gathered} .
$$

4. Translation and Modulation.

$$
\left[Z\left(M_{2 \pi b} T_{n} f\right)\right](t, \omega)=e^{2 \pi i(m t-n \omega)} \tilde{f}(t, \omega) .
$$

5. conjugation.

$$
[z(\bar{f})](t, \omega)=\overline{\tilde{f}(t,-\omega)}
$$

6. Symmetric.

(a). If $f$ is even function, then

$$
(Z f)(t, \omega)=\tilde{f}(-t,-\omega)
$$

(b). If $f$ is odd function, then

$$
(Z f)(t, \omega)=-\tilde{f}(-t,-\omega) .
$$

7. Inversion. 


\section{Asriadi $^{1}$, Budi Nurwahyu ${ }^{2}$, Mawardi Bahri ${ }^{3}$}

$$
\begin{gathered}
f(t)=\int_{0}^{1} \tilde{f}(t, \omega) d \omega \\
\hat{f}(\omega)=\int_{0}^{1} e^{-2 \pi i \omega t} \tilde{f}(t, \omega) d t \\
f(\omega)=\int_{0}^{1} e^{-2 \pi i \omega t} \tilde{f}(t, \omega) d t
\end{gathered}
$$

8. Dilatation.

$$
\left(z D_{\frac{1}{a}} f\right)(t, \omega)=\tilde{f}\left(a t, \frac{\omega}{a}\right)
$$

9. Convolution.

$$
\tilde{f}(t, \omega) \overline{\tilde{f}(t, \omega)}=\sum_{m, n=-\infty}^{\infty}\left\langle f, M_{2 \pi m} T_{-n} g\right\rangle e^{2 \pi i(m t+n \omega)} .
$$

\section{Teorema 1.2 [3]}

Zak transformation is a symmetric mapping of $L^{2}(\mathbb{R})$ ke $L^{2}\left([0,1]^{2}\right)$.

In 1970 Linear Canonic Transformation (LCT) was firstly proposed by Moshinsky and Collins [5]. Next, the definition of LCT as follows

\section{Definisi1.2 [2]}

Let $A=(a, b, c, d)=\left[\begin{array}{ll}a & b \\ c & d\end{array}\right] \in \mathbb{R}^{2 \times 2}$ be a matrix of parameter that satisfies $\operatorname{det}(A)=a d-$ $b c=1$, LCT of a signal $f(t) \in L^{1}(\mathbb{R})$ be given as

$$
\mathcal{L}_{A}\{f\}(\omega)=\left\{\begin{array}{c}
\int_{-\infty}^{\infty} f(t) K_{A}(\omega, t) d t, \quad b \neq 0 \\
\sqrt{d} e^{i \frac{c d}{2} \omega^{2}} f(d \omega), b=0
\end{array}\right.
$$

Where $K_{A}(\omega, t)$ is kernel of LCT that is given as follows

$$
K_{A}(\omega, t)=\frac{1}{\sqrt{2 \pi b i}} e^{i \frac{1}{2}\left(\frac{a}{b} t^{2}-\frac{2}{b} t \omega+\frac{d}{b} \omega^{2}\right)} .
$$

It is easy to show that kernel of LCT satisfies the following properties

$$
K_{A^{-1}}(\omega, t)=\frac{1}{\sqrt{-2 \pi b i}} e^{-i \frac{1}{2}\left(\frac{a}{b} t^{2}-\frac{2}{b} t \omega+\frac{d}{b} \omega^{2}\right)} .
$$

From definition 1.2, it obvious that if $b=0$ then LCT is a product of $\sqrt{d} e^{i \frac{c d}{2} \omega^{2}}$ and $d \omega$ Therefore, we only use for $b \neq 0$.

In the particularly case, if $A=(0,1,-1,0)$ then definition of LCT can be derived to be Fourier transformation, namely

$$
\mathcal{L}_{A}\{f\}(\omega)=\mathcal{F}_{A}\{f\}(\omega)=\frac{1}{\sqrt{2 \pi}} \int_{-\infty}^{\infty} f(x) e^{-i t \omega} d t
$$




\section{Asriadi $^{1}$, Budi Nurwahyu ${ }^{2}$, Mawardi Bahri ${ }^{3}$}

\section{Teorema1.3 [3]}

Let $f, g \in L^{1}(\mathbb{R})$ then the following properties hold

1. Translation.

For all real number $k$ then

2. Modulation.

$$
\mathcal{L}_{A}\left\{\tau_{k} f\right\}(\omega)=\mathcal{L}_{A}\{f(t-k)\}(\omega)=e^{\frac{-i a c k^{2}}{2}+i c k \omega} \mathcal{F}_{A}(\omega-a k) .
$$

For all real number $\omega_{0}$ then

$$
\mathcal{L}_{A}\left\{\boldsymbol{M}_{\omega_{0}} f\right\}(\omega)=\mathcal{L}_{A}\left\{e^{i t \omega_{0}} f(t)\right\}(\omega)=e^{-\frac{i b d \omega_{0}^{2}}{2}+i d \omega_{0} \omega_{\mathcal{F}}} \mathcal{F}_{A}\left(\omega-b \omega_{0}\right) .
$$

3. Translation and modulation.

For all real number $k$ and $\omega_{0}$ then

$$
\mathcal{L}_{A}\left\{\boldsymbol{M}_{\omega_{0}} \tau_{k} f\right\}(\omega)=\mathcal{L}_{A}\left\{e^{i x \omega_{0}} f(x-k)\right\}(\omega) .
$$

\section{Linear canonical Zak transformation}

Liner Canonical Zak Transformation (LCZT) firstly was introduced by Bahri and Ashino

Definition 2.1[1]

LCZT of a signal $f \in L^{2}(\mathbb{R})$ at point $(t, \omega) \in \mathbb{R} \times \mathbb{R}$ is

$$
\left(Z_{A} f\right)(x, \omega)=\frac{1}{\sqrt{2 \pi b i}} \sum_{k \in \mathbb{Z}} f(t-k) e^{i \pi\left(\frac{a}{b} k^{2}-\frac{2}{b} k \omega+\frac{d}{b} \omega^{2}\right)} .
$$

Where $A=(a, b, c, d)=\left[\begin{array}{ll}a & b \\ c & d\end{array}\right] \in \mathbb{R}^{2 \times 2}$ is a parameter matrix and

If we choose $A_{Z}=(0,1,-1,0)$ then LCZT can be derived to become definition of Zak transformation with multiplier $\frac{1}{\sqrt{2 \pi i}}$ as follows

$$
\left(Z_{A_{Z}} f\right)(x, \omega)=\frac{1}{\sqrt{2 \pi i}} \sum_{k \in \mathbb{Z}} f(t-k) e^{2 k \omega \pi i}
$$

Remarks: If from kernel LCT $\frac{1}{\sqrt{2 \pi b i}} e^{i \frac{1}{2}\left(\frac{a}{b} t^{2}-\frac{2}{b} t \omega+\frac{d}{b} \omega^{2}\right)}$ multiplier $\frac{1}{2}$ replaced by $\pi$ and variable $t$ replaced by $k$, then we obtain kernel of LCZT, namely $\frac{1}{\sqrt{2 \pi b i}} e^{i \pi\left(\frac{a}{b} k^{2}-\frac{2}{b} k \omega+\frac{d}{b} \omega^{2}\right)}$.

Theorem 2.2 (linear property).

If $f, g \in L^{2}(\mathbb{R})$ and let $A=(a, b, c, d)=\left[\begin{array}{ll}a & b \\ c & d\end{array}\right] \in \mathbb{R}^{2 \times 2}$, then for all $\alpha, \beta \in \mathbb{C}$

$$
Z_{A}(\alpha f+\beta g)(t, \omega)=\alpha Z_{A} f(t, \omega)+\beta Z_{A} g(t, \omega)
$$

Proof.

From definition 2.1 we have 


\section{Asriadi $^{1}$, Budi Nurwahyu ${ }^{2}$, Mawardi Bahri ${ }^{3}$}

$$
\begin{aligned}
& Z_{A}(\alpha f+\beta g)(t, \omega) \\
& =\frac{1}{\sqrt{2 \pi b i}} \sum_{k \in \mathbb{Z}}(\alpha f+\beta g)(t-k) e^{\frac{i \pi}{b}\left(a k^{2}-2 k \omega+d \omega^{2}\right)} \\
& =\frac{1}{\sqrt{2 \pi b i}} \sum_{k \in \mathbb{Z}}(\alpha f(t-k)+\beta g(t-k)) e^{\frac{i \pi}{b}\left(a k^{2}-2 k \omega+d \omega^{2}\right)} \\
& =\frac{1}{\sqrt{2 \pi b i}} \sum_{k \in \mathbb{Z}}\left[\alpha f(t-k) e^{\frac{i \pi}{b}\left(a k^{2}-2 k \omega+d \omega^{2}\right)}+\beta g(t-k) e^{\frac{i \pi}{b}\left(a k^{2}-2 k \omega+d \omega^{2}\right)}\right] \\
& =\alpha \frac{1}{\sqrt{2 \pi b i}} \sum_{k \in \mathbb{Z}} f(t-k) e^{\frac{i \pi}{b}\left(a k^{2}-2 k \omega+d \omega^{2}\right)}+\beta \frac{1}{\sqrt{2 \pi b i}} \sum_{k \in \mathbb{Z}} g(t-k) e^{\frac{i \pi}{b}\left(a k^{2}-2 k \omega+d \omega^{2}\right)} \\
& =\alpha Z_{A} f(t, \omega)+\beta Z_{A} g(t, \omega) .
\end{aligned}
$$

Theorem 2.3 (translation property).

If $\in L^{2}(\mathbb{R})$ and $A=(a, b, c, d)=\left[\begin{array}{ll}a & b \\ c & d\end{array}\right] \in \mathbb{R}^{2 \times 2}$, then for all $m \in \mathbb{Z}$

where $T_{m} f(t)=f(t-m)$.

$$
Z_{A}\left(T_{m} f\right)(t, \omega)=e^{-\pi i c\left(2 m \omega+c m^{2}\right)} Z_{A} f(t, \omega+a m)
$$

Proof.

Claim that

$$
Z_{A}\left(T_{m} f\right)(t, \omega)=e^{-\pi i c\left(2 m \omega+c m^{2}\right)} Z_{A} f(t, \omega+a m) .
$$

From definition2., we obtain

$$
\begin{aligned}
& Z_{A}\left(T_{m} f\right)(x, \omega) \\
& =\frac{1}{\sqrt{2 \pi b i}} \sum_{k \in \mathbb{Z}} f((t-k)-m) e^{\frac{i \pi}{b}\left(a k^{2}-2 k \omega+d \omega^{2}\right)} \\
& =\frac{1}{\sqrt{2 \pi b i}} \sum_{k \in \mathbb{Z}} f(t-(k+m)) e^{\frac{i \pi}{b}\left(a((k+m)-m)^{2}-2((k+m)-m) \omega+d \omega^{2}\right)} \\
& =\frac{1}{\sqrt{2 \pi b i}} \sum_{k \in \mathbb{Z}} f(t-k) e^{\frac{i \pi}{b}\left(a(k-m)^{2}-2(k-m) \omega+d \omega^{2}\right)} \\
& =\frac{1}{\sqrt{2 \pi b i}} \sum_{l \in \mathbb{Z}} f(t-l) e^{\frac{i \pi}{b}\left(a\left(l-2 m l+m^{2}\right)-2(l-m) \omega+d \omega^{2}\right)} \\
& =\frac{1}{\sqrt{2 \pi b i}} \sum_{l \in \mathbb{Z}} f(t-l) e^{\frac{i \pi}{b}\left(a l^{2}-2 a m l+a m^{2}-2 l \omega+2 m \omega+d \omega^{2}\right)} \\
& =\frac{1}{\sqrt{2 \pi b i}} \sum_{l \in \mathbb{Z}} f(t-l) e^{\frac{i \pi}{b}\left(a l^{2}-2 l(\omega+a m)+d(\omega+a m)^{2}-2 d a m \omega-d a^{2} m^{2}+a m^{2}+2 m \omega\right)} \\
& =\frac{1}{\sqrt{2 \pi b i}} \sum_{l \in \mathbb{Z}} f(t-l) e^{\frac{i}{2 b}\left(a l^{2}-2 l(\omega+a m)+d(\omega+a m)^{2}\right)} e^{\frac{i \pi}{b}\left(-2 d a m \omega-d a^{2} m^{2}+a m^{2}+2 m \omega\right)} \\
& =\frac{1}{\sqrt{2 \pi b i}} \sum_{l \in \mathbb{Z}} f(t-l) e^{\frac{i \pi}{b}\left(a l^{2}-2 l(\omega+a m)+d(\omega+a m)^{2}\right)} e^{\frac{i \pi}{b}\left(-2 d a m \omega-d a^{2} m^{2}+a m^{2}+2 m \omega\right)}
\end{aligned}
$$




\section{Asriadi $^{1}$, Budi Nurwahyu ${ }^{2}$, Mawardi Bahri ${ }^{3}$}

$$
\begin{aligned}
& =e^{\frac{i \pi}{b}\left(-2 d a m \omega-d a^{2} m^{2}+a m^{2}+2 m \omega\right)}\left(\frac{1}{\sqrt{2 \pi b i}} \sum_{l \in \mathbb{Z}} f(t-l) e^{\frac{i \pi}{b}\left(a l^{2}-2 l(\omega+a m)+d(\omega+a m)^{2}\right)}\right) \\
& =e^{\frac{i \pi}{b}\left(-2 d a m \omega-d a^{2} m^{2}+a m^{2}+2 m \omega\right)} Z_{A} f(t, \omega+a m) \\
& =e^{\frac{i \pi}{b}(-2 d a m \omega+2 m \omega)} e^{\frac{i \pi}{b}\left(-d a^{2} m^{2}+a m^{2}\right)} Z_{A} f(t, \omega+a m) \\
& =e^{-2 i \pi\left(\frac{d a-1}{b}\right) m \omega} e^{-i \pi\left(\frac{d a-1}{b}\right) m^{2}} Z_{A} f(t, \omega+a m) \\
& =e^{-2 \pi i\left(\frac{d a-1}{b}\right) m \omega} e^{-i \pi\left(\frac{d a-1}{b}\right) m^{2}} Z_{A} f(t, \omega+a m) .
\end{aligned}
$$

Since $a d-b c=1$ then $\frac{d a-1}{b}=c$, thus we have

$Z_{A}\left(T_{m} f\right)(t, \omega)=e^{-2 \pi i c m \omega} e^{-i \pi c m^{2}} Z_{A} f(t, \omega+a m)=e^{-\pi i c\left(2 m \omega+c m^{2}\right)} Z_{A} f(t, \omega+a m)$.

\section{Conclusion}

In LCZT if $f, g \in L^{2}(\mathbb{R})$ and $A=(a, b, c, d)=\left[\begin{array}{ll}a & b \\ c & d\end{array}\right] \in \mathbb{R}^{2 \times 2}$ then for all $\alpha, \beta \in \mathbb{C}$ we get

$$
Z_{A}(\alpha f+\beta g)(t, \omega)=\alpha Z_{A} f(t, \omega)+\beta Z_{A} g(t, \omega),
$$

and for translation property in LCZT, if $f \in L^{2}(\mathbb{R})$ and $A=(a, b, c, d)=\left[\begin{array}{ll}a & b \\ c & d\end{array}\right] \in \mathbb{R}^{2 \times 2}$ then for all $m \in \mathbb{Z}$ hold

$$
Z_{A}\left(T_{m} f\right)(t, \omega)=e^{-\pi i c\left(2 m \omega+c m^{2}\right)} Z_{A} f(t, \omega+a m)
$$

\section{References}

[1]. Bahri, M., and Ashino, R., 2016. Logarithmic uncertainty principle for quaternion linear canonical transform. In Wavelet Analysis and Pattern Recognition (ICWAPR),IEEE International Conference on (pp. 140-145).

[2]. Bahri, M., Amir, A. K., and Ashino, R., 2017. Correlation formulation using relationship between convolution and correlation in linear canonical transform domain. In Wavelet Analysis and Pattern Recognition (ICWAPR), International Conference on (pp. 177-182). IEEE.

[3]. Lokenath Debnath and Shah,F.A., 2015. Wavelet Transforms and Their Applications, second edition. Birkhauser, Boston. 268-283.

[4]. Zhang, Q., 2016. Zak transform and uncertainty principles associated with the linear canonical transform. IET Signal Processing, 10(7), 791-797.

[5]. Bastiaans, M. J. and Alieva, T., 2016. The linear canonical transformation: definition and properties. In Linear Canonical Transforms (pp. 29-80). Springer, New York, NY. 